\title{
Ultrasonografía del cráneo por fontanela anterior en recién nacidos lactantes
}

\author{
Dr. lirnesto Grob 13.1
}

\author{
Cranial ul trasound exploration in infants
}

\begin{abstract}
Cranial ultrasound through the anterior fontancle in infants allows a wide view of the different brain structures, both normal or pathologic: 23 ultrasonic exaninations were carried out in 20 infant paticnts, most of thern newborns, with perinatal hypoxia, prematurify, infections of the central nervous system, craniai abmimalities or trauma and neuromuscular diseases. Our results confirm the importance of cranial ultrasound through the antetior fontanclic, because it is a simple, relatively unexpensive techniljue to perform, allowing tarly anatomic evaluation of highrisk patients and follow up of intants with entrul nerrous systcm diseuses.

(Key words: cranial ultrasound, infänts, diagnosis, brain injurios, perinatal hypoxia, central nervous system infections).
\end{abstract}

Las pritneras aplicaciones clínicas del ultrasonido fueron realizadas lace más de veinte años. Durante mucho tiempo el Modo A constituyó cl único nétodo ultrasónico para deterininar la linea media y efectuar mediciones de los ventrículos cercbrales. A partir de 1968 es notorio el progreso en esta área, con lat implementación de técnicas tales como: Modo-B, que permite a Sjörgren y Lombroso ${ }^{12}$ obtener una idea de la morfología de los ventrículos cerebrales; escata de gris, que posibilita a Kossof ${ }^{3}$ describir con precisión la anatomia ultrasonográfica del cerebro normal en el niño; Johnson y Morgan ${ }^{4,5}$, mediante cortes axiales transóseos logran intercsantes correlaciones con la tomografía.

Sin embargo, debido a la interfasc hueso-tejido, las técnicas transóseas sun incompatibles con un análisis detallado del parénquima cerebral, situación que es ampliamente superada al utilizar la via transfontanelar para la exploración ultrasónica del cerebro.

Ber-Ora y Allan ${ }^{6.7}$ cn USA y Couture ${ }^{8}$ en Francia son los primeros en utilizar esta fécnica y destacar la posibilidad de lograr una visión panorárnica del contenido intracraneano, permitiendo visualizar no sólo las cavidades ventriculares sino también el parénquima cerebrail.

E] presente trabajo pretende mostrar nuestray experiencia en ultrasonografia cercbral transfontanela anterior y su importancia en el niño con riesgo cerebrat.

1. Unidad de neonatologia. Servicio de pediatría, Hospital Regional de Osorno.

\section{MATERIAL Y METODO}

Las ultrawnografías cetcbrales se efectuaron mediante un Ultrasonógrato ALOKA SSD 256 A T'iempo Real con transductor lineal de $3,5 \mathrm{MHZ}$. Se realizaroul los exámenes sin premedicación del paciente, utilizando la técrica transfontanela anterior ${ }^{9}$. La explnración consta de dos series de cortes: coronales foblicuo anterior, medio posterior): sagitales (agital medio y paragitales oblicuos en el eje de cada ventrículo lateral) Mediante esta técnica se explora la casi totalidad del sistema ventricular, núcleos de la base, parencquina wrebral, fosa posterior y para visualizar coleccjones peri-ccrebrales se interpone un medio transónico.

Comalmente los cortes por la fontancla anterior permiten, en primer tórmino, reconocer las referencias oscal 3, cuerpo del estenoides y hueso occipital. I.l siste ma ventricular aparece como Iransónico dado su contenido licyuido. Los plexos coroideos tienen un aspecto característico lineal y muy ecogénicos, dada la riqueza de su vascularización. El parénquima ccrebral presenta una eco-estructura homogénea, sobrc la cual se visuálizan líncas onduladas muy ecogénicas, que corresponden a las circunvoluciones cerebrales. La región de los núcleos grises centrales (tálamos, núclcos caudados) son fácilmente reconocibles por su forma rodondcada y su carácter hiper-ecogénico 9,10 .

Sc practicaron cstas expluraciones en el períado comprendidu entre septiembre de 1985 y abril de 1986 , realizándose 23 ultrasonografíaz cercbralcs a 20 pacientos pediátricos provenientes del Servicio de Neonatolo. gía y Pediatría del Hospital Regional de Osorno y de la Clínica Alemana de Osorno, en los cuales existía indicación de exploración cerebral.

\section{RESULTADOS}

En la Tabla I se muestran las indicaciones que motivaron las exploraciones ultrasonográlicas transfontanela anterior, en ésta se aprecia que la mayoría de ellas corresponden a situaciones de 
riesgo cerebral potencial, destacando la hipoxia y la prematuridad entre ellas.

El resultado obterido en las veintitrés ultrasonografías cerebrales realizadas a veinte pacientes se aprecid en la Tabla $2: 41,6 \%$ se consideraron normales $y$ en $58,4 \%$ se cncontraron alteraciones, entre las cuales destaca la dilatación vent ricular.

A continuación se describen las situaciones clínicas que llevaron a esta explaracion y las anormalidades encontradas (Tabla 3):

Hipoxia: en nuestra casuística corresponde al $33.3 \%$ de las exploraciones cerebrales. siendu etl $55.5 \%$ normales $y$ en $44.5 \%$ patológicas cniontrándose diferentes lesiones que se devallan en lit misria $1 \mathrm{abla.}$

Prematuriad: constituye la segunda situdión disnica mis freouente que llevo a una exploracion ultrasonica. encontrándose en la mitad de ellas altoraciones coográficas cotno: hembragia cere. bral, alteracion de la homogeneidad fe la eco. estrustura cerebral, porencelidia fromtal, coleccion peri-cerebral (tigura ! a).

Alteración del perimetro craneano: an nues1rat experiencia constituje. al igual que la anterior. una situación clínica lirecultute que llevó a la ultrasomogratía cerebral. encontrándose alteraciores en la rrilad de slas: un caso de dilatacion ventricular $y$ purencefalia froutal y otro pitciente con una colección pericerebral (figura 1 b).

Infeccion det Sistema Verioso Central: corresponde a la cuarta indicación de exploración ccrebral y en la cual aparecen el mayor múmero de alteraciones. Todos los casos explorados presentaban alteraciones, siendo la mas trecuente lá dilatación tri o bi-ventricular. pudietrdo traducir una hidronefalia por bloyueo o una atrofia corti. cal (figura 2). En un laciante encontramos imágenes ecogénicas intraventriculares sospechosas de una ventriculitis (figura l b y c) $y$ en otro paciente una colección peri-cerebral moderada (figura $1 \mathrm{~b}$ ).

Malformaciones: Se realizarot tres exploraciones: la primera correspionde a un recien nacido portadur de una focomelia. en el cual no encontramos alteraciones ecogtáticas: dos iasos con malformaciones cerebrales: una nalformación de Arnold Chiari Tipo Il (figura 3 a) en un recién nacido portador de ura mielomeningocele lumbar y un recièn nacido con una hidranebcefalia (figura 3 b).

Ventilacion mecanica: la exploración ultrasónica en un recién nacido fue normal y en utro pacicnte se observó una hemorragia sub-ependimaria (figura 3 c) $y$ alteración de la homogeneidad de la ecoestrustura cerebral.

Traumotismo crancano: en un lactante de ocho meses portador de un sindrome del niño
Tabla 1.

Problemas elínroos que motivaron la indicación de 23 ultrasonografías curebrales en 20 lactanses

\begin{tabular}{|c|c|c|}
\hline Problema & $n$ & $\%$ \\
\hline Hipoxia peri-natal & 9 & 33,3 \\
\hline Prematuridad & 4 & ] 4,8 \\
\hline $\begin{array}{l}\text { Alteración del crecimjento } \\
\text { craneano }\end{array}$ & 4 & 14,8 \\
\hline Infecciones del SNC & 3 & 11,1 \\
\hline Malf ormaciones & 3 & 11,1 \\
\hline Ventilación asistida & 2 & 7.4 \\
\hline Traumatismo craneano & 1 & 3.7 \\
\hline Nutcración neuro-rnuscular & 1 & 3,7 \\
\hline TOTAL. DI:L PROBLIMA & $27\left(^{*}\right)$ & 100 \\
\hline
\end{tabular}

\{ $^{*}$ Se consjueró Jnás de un problerna por paciente.

Tabla 2.

Lesiones jdentificadas en 23 u]trasonomeratias cerebrales

\begin{tabular}{lcr}
\hline Lesion & $\mathrm{n}$ & $\%$ \\
\hline Dilatación ventricular & 5 & 10,8 \\
Hemorragía cerebral & 2 & 8,3 \\
Colección peri-natal & 2 & 8,3 \\
Maltormación cerebral & 2 & 8,3 \\
Porencetalia & 1 & 4,2 \\
Ventriculitis & 1 & 4,2 \\
Alteración cn la homogenejdad & 1 & \\
de 1a eco-estructurá cerebral & {$[0$} & 41,2 \\
Normal & $24(*)$ & 100 \\
\hline TOTAL & & \\
\hline
\end{tabular}

(*) Vatios pacientes presentaban más de unir lesión

golpeado, con lracturas múltiples de cráneo, se observó una hemotragia parenquinatosa fruntal (figura $3 \mathrm{~d}$ ).

1/teracion neurontiscular: sc procedio a una exploración cerebral en un recién nacido portador de un síndrome de hipotonía (floppy baby). siendo esta nomal.

\section{COMENTARIO}

El aporte extraordinario de la ultrasonografía transfontanela anterior etl el nitio con riesgo cerebral ha sido demostrado en múltiples publicaciones1-14. Esta [écnica asegura una visión panorímica del sistema ventricular, de los hemisterios cercbrales, núcleos de la base y fosa posterior. Permite una visión completa delsistema ventricular, no solamenie de los ventriculos loterales sino rambien del tereer y cuarto ven. trículos $y$ cisterna de la base. $\Lambda$ demás nos fá una visión del parénquina aerebral: anomalias de estructura, presencia de masas o colecciones de dilicrente naturaleza ${ }^{15}$. 

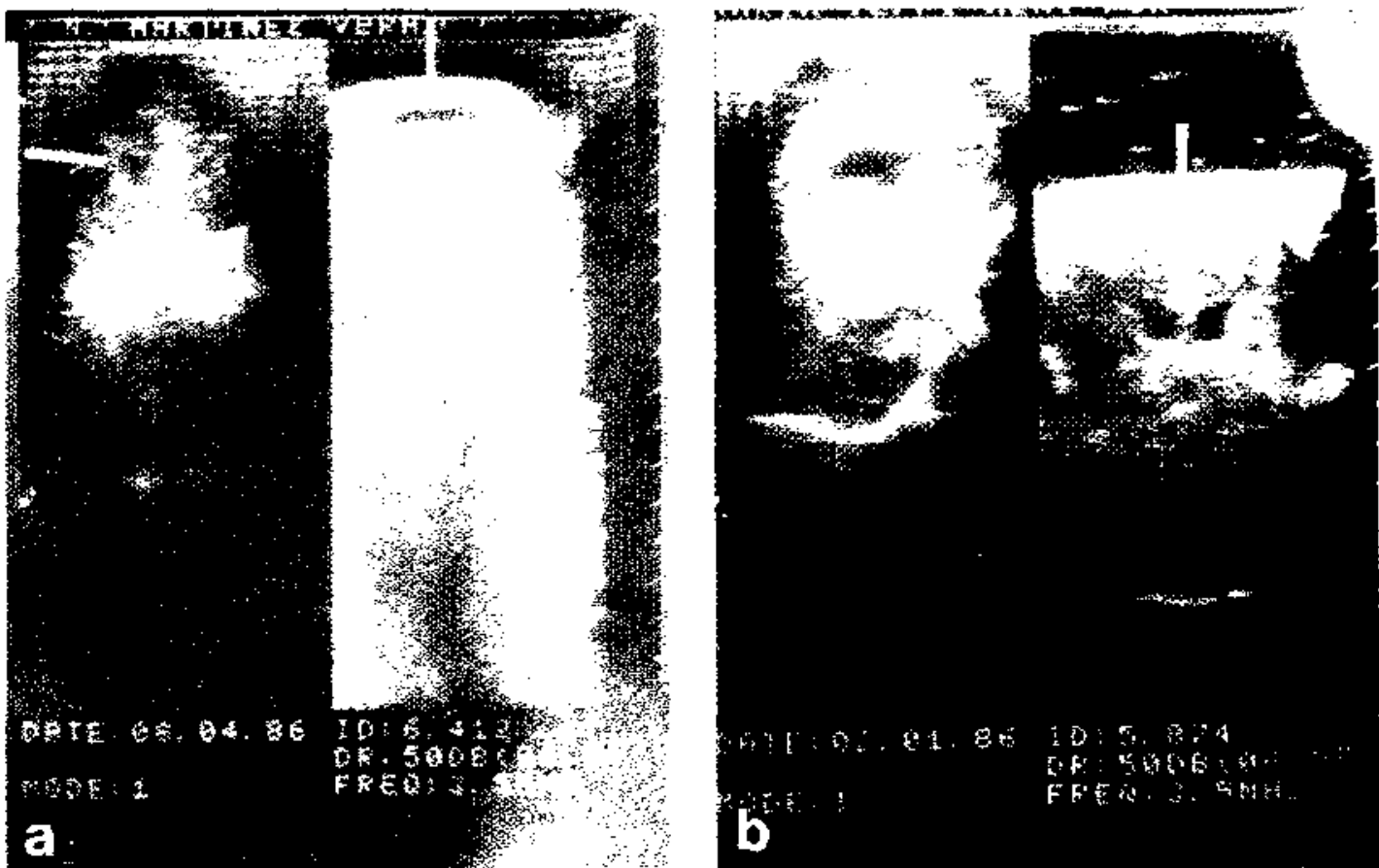

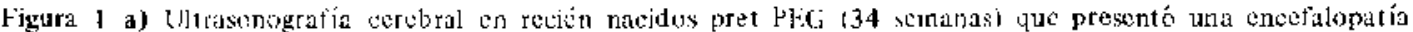

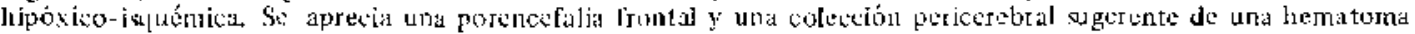
sub-dural. b) Lactanti de 4 meses portador de una metningitis purulenta en que la ultrásonografía demuestra una aleracion al al tamatio g torma de los ventrículus laterales compatibles con atrofia cortical $y$ una colección peri-crebral.
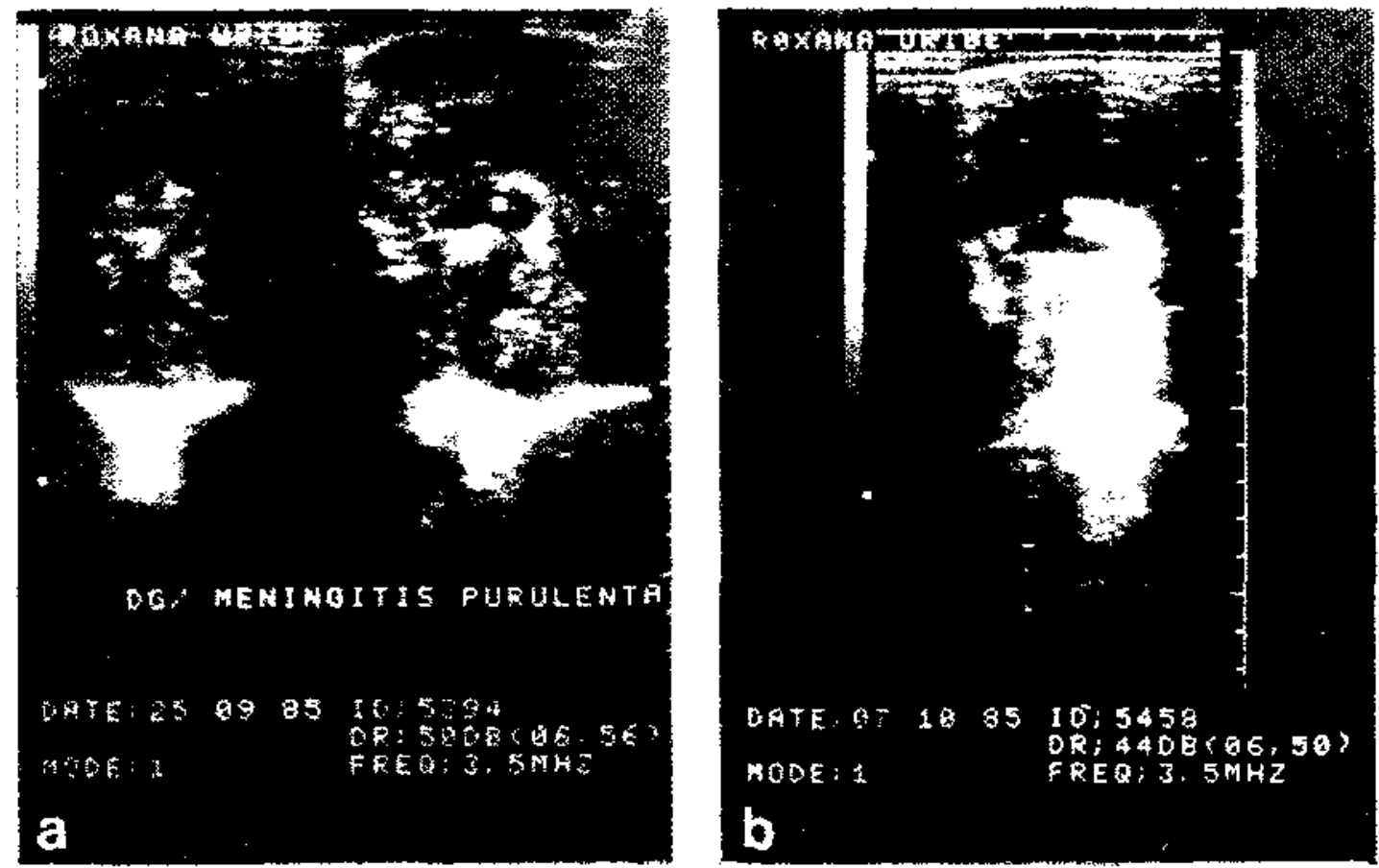

Figura 2. Lactarte de 5 meses portador de una meningitis purulenta de evolucjón grave en la cual la ultrasonografía revela una dilatación tri-ventricular: a) Inágenes ecogénicas intraventriculares sospechosas de ventriculitis que crolucionan hacia la progresión de una dilatación bi-ventricular b) Imagen compatible con dilatación bi-ventricular. 

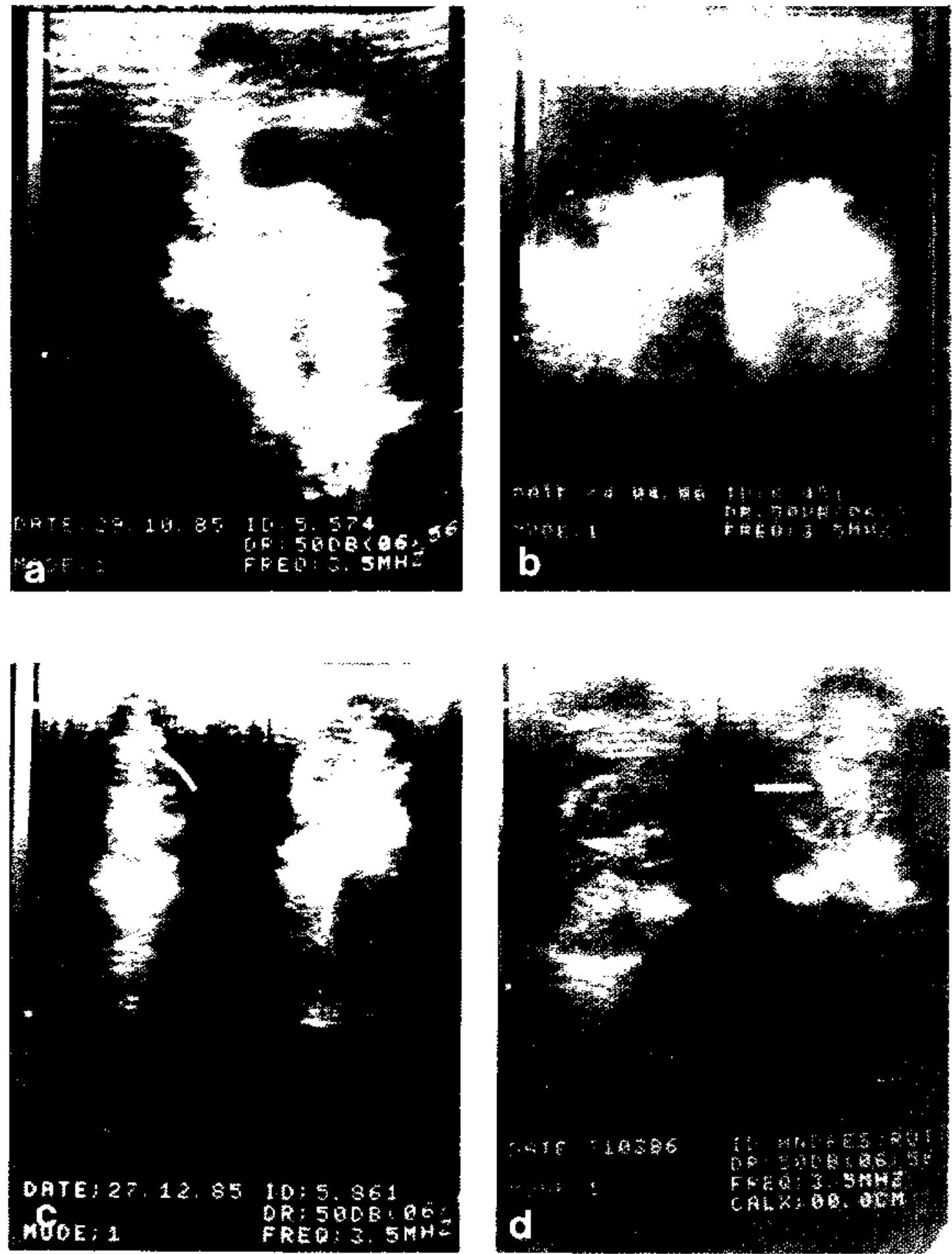

Figura 3 a) Ultrasonogratia cerebral en un recién nacido portadot de un mielo-meningocele lumbar, en que se aprecid una dilatación bi-ventricular stvera y signos sugcrentes de una maltormación de Annold-Chiari tipo lI. b) La ultrasonogralía cerebral demuestra en los cortes sagital y coronal la presencia de líquido intracraneano con ausencir de parénquima terebral compatibles con una hidranencefalia. c) Ultrasonogratia cerebral en un prematuro extremo que requirió ventilación asistida en que se obscrva una pequeña hemorragia sub-ependimaria que evoluciona hacia la regresión total. d) La ultrasonugrafía cerebral en un lactante de b meses con un sindrome de Silverman revelin uma lemiorragia parenquimatosa lironto-talámica derecha. 
Tahla 3.

Anlomal ías cerebrales detecladas por ultrasonografia en 20 lactantes segun problema clínico

\begin{tabular}{|c|c|c|c|}
\hline Problemas & $\begin{array}{l}\text { Pacientes } \\
\text { (n) }\end{array}$ & & $\begin{array}{c}\text { Altcración } \\
\text { (nj/Anormales) }\end{array}$ \\
\hline Hipox ia peri-natat & 9 & & \\
\hline Notmales & 5 & & \\
\hline \multirow[t]{5}{*}{ Anormales } & 4 & & \\
\hline & & $\begin{array}{l}\text { - Dilatación ventricular } \\
\text { - Ilemorraeia cerebral }\end{array}$ & $\begin{array}{l}2 / 4 \\
1 / 4\end{array}$ \\
\hline & & Colecrion perj-corebral & $1 / 4$ \\
\hline & & - Alteración homogeneidad & \\
\hline & 4 & & $1 / 4$ \\
\hline \multirow{6}{*}{$\begin{array}{c}\text { Prematuridad } \\
\text { Nornales } \\
\text { Anoimales }\end{array}$} & $\begin{array}{l}4 \\
2\end{array}$ & & \\
\hline & 2 & & \\
\hline & & Hemortagia cerebtal & $1 / 2$ \\
\hline & & $\therefore$ Porencetalia & $1 / 2$ \\
\hline & & - Colección peri-cerebral & $1 / 2$ \\
\hline & & $\begin{array}{l}\text { Alteración homogeneidad } \\
\text { eco-estructura cerebral }\end{array}$ & $1 / 2$ \\
\hline Alteración Crec. Craneano & 4 & & \\
\hline Normales & 2 & & \\
\hline \multirow[t]{4}{*}{ Anormales } & 2 & & \\
\hline & & - Dilatación ventricular & $1 / 2$ \\
\hline & & - Colccción peri-cerebral & $1 / 2$ \\
\hline & & Ротелcefalia & $1 / 2$ \\
\hline Intecciones SNC & 3 & & \\
\hline Normales & 0 & & \\
\hline \multirow[t]{4}{*}{ Anormales } & 3 & & \\
\hline & & - Dílatación ventricular & $3 / 3$ \\
\hline & & - Ventriculitix & $1 / 3$ \\
\hline & & Colección peri-cetelral & $1 / 3$ \\
\hline Maltormaciones & 3 & & \\
\hline Normales & 1 & & \\
\hline \multirow[t]{2}{*}{ Anormales } & 2 & & \\
\hline & & - Anencefalia & $1 / 2$ \\
\hline Ventilación asistida & 3 & - Arnold-Chiari & \\
\hline Normales & 1 & & \\
\hline \multirow[t]{3}{*}{ Anormales } & 2 & & \\
\hline & & Ifemortagia cerebral & $1 / 2$ \\
\hline & & $\begin{array}{l}\text { - Alteración homogeneidad } \\
\text { eco-estructura cerebral }\end{array}$ & $1 / 2$ \\
\hline Traumatismo craneano & 1 & & \\
\hline Normales & 0 & & \\
\hline \multirow[t]{2}{*}{ A normales } & $i$ & & \\
\hline & & - Hemonagia cerebral & $1 / 1$ \\
\hline Ajteración neuro-muscular & 1 & & \\
\hline Normales & 1 & & \\
\hline Anormales & 0 & & $0 / 0$ \\
\hline
\end{tabular}

La exploración anatómica cubre prácticamente todo el cerebro, desde los lóbulos frontales hasta los occipitales. El comportamiento ultrasónico de las diferentes estructuras cerebrales permite reconocerlas. como ser los plexos coroideos y cl vermix cerebeloso. La interfase de sustancia gris-blanca se visualiza a nivel del tálamo y la cabezád del núcleo caudado 16,17 .

Todas estas ventajas le otorgan un lugar de privilegio al ultrasonido, dentro de la exploración neuro-radiológica. dada su facilidad de realización, inocuidad, costo, etc. No obstante, la ultrasonografía cerebral presenta algunos inconvenien. tes, tales como su limitación en el tiempo por el cierre de la fontanela anterior, impidiendo su aplicación después del año de edad. Así también, puede presentar poca especificidad de las imágenes $y$ algunos procesos como la isquemia o el edema no pueden ser perfectamente diferenciadus 9 .

La utilidad de esta técnica es evidente y destacada en el periodo neonatal, dada la alta incidencia de accidentes neurológicos ${ }^{11}$. En este grupo etario, como nosotros lo describimos, existen dos situaciones que hacen de esta técnica un examen indispensable, tales como la prema- 
turidad $y$ el sufrimiento fetal hipóxicot. Ė estos niños la exploración ultrasónica nos aporta una referencia inmediata respecto a una lesión cerebral frecuente en el prematuro, como es la hetnorragia cerebrai 20.25 ; constituyendo una de las principalts causas de mortalidad y morbilidad cn este grupo etario. Varios investigadores como Allan, Bejar, Couke, ete. constatal frecuencias variables de lesiones hemorrágicas cerebrales que van desde un 27 a un $90 \%$ dependicndo de la ciad gestacional ${ }^{26-30}$.

Otra indicación importante de exploración cerebrai, como sc confirmó en nuestra experiencia, corresponde al diagnóstico de lás maliormaciones cerebrales ${ }^{31-35}$. El ultrasonido debería ser el examen de primera intención en esia patologia, siendo suficiente para el diagnóstico de varias de ellas, tales como la holoprosencefatia, hidridnencefalia. agenesia del cuerpo caloso, sindrome đe Dandy.Walker y mús alún pará el diagnóstion ante-natal de estas lesiones $36,37,38$.

Un lugar destacado de la ultrasonugrafía cerebra] en nucstra experiencia corresponde a la cvaluacion de las infectiones Je] sistema netrioso centrul, frecuente en nuestro medio. En ellas observamos una al ta frecuencia de alteraciones y permite el diagnóstico precoz de algunas complicationes. como también hia sido descrito por otros autores $9,33,35,39,40$. Las infecciones bacterianas meningeas en cl lictante pueden complicarse y dar origen a bloqueos de los espacios de reabsorción periaracnoídeos. llevando a una hidrocefalia comunicante pesquisable por ultrasonografía. Además. Couture ${ }^{9}$ describe imagenes ecogénicas intraventriculares lineales como ma. nifestación de un ventriculitis; un efecto de "masa" desde el punto de vista ecográfico que traduce una colección intraparenguimatosa como en el abseso cerebral. y por último una imagen anccogénica pericerebral que traduce una colección sub-dural o sub-aracnoidea.

En conclusión, el futuro de la ultrasonogralía cerebral transfontaneda anterior es fácilmente previsible en las unidades de neonatologia $y$ pediatria. aportando un servicio insospechado por su facilidad de realización, inocuidda y costo. permiliendo el diagnóstico oportuno y adecuado de patologias y complicaciones del sistema nervioso central.

\section{RESUMEN}

La ultrasonografía cerebral por vía transfonta. nela anterior es und técrica neuroradiológica moderna que permite una visión panorámica de las diferentes estructuras normales y patológicas del cerebro.

Se realizaron 23 ultrasonografías cerebrales a
20 pacientes pediátricos, en su mayoría recién nacidos, en los cuales existía indicación de exploración cerebral. Destacan por su frecuencia la hipoxia peri-natal y la prematuridad. siendo en las infecciones del sistema nervioso central donde se observó la mayor severidad de las lesiones.

En conclusión, los resultados confìrman la importancia de la ultrasonugrafóa cerebral transfontanela anterior en el nimo con riesgo cercbral en nuestro medio, dada su facilidad de realización. inocuidad y costo, permitiendo el diagnóstico opurtuno y adecuado de patologías y complicaciones del sistema nervioso central.

\section{REFERENCIAS}

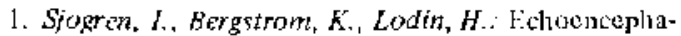
lograplyy in infants and childten. Acta Radiol (Suppl) 278: 7, $196 \mathrm{~B}$.

2. Lombroso, C.T., Erbo, G., Yogo, $T$, logowitz, $V_{\text {.: }}$ Two dimensional ultrasonograpiny: a method to study normal and abmotrnal ventricic. Pediatrics 42 157,1968

3. Kossof, G, Garret, W.J., Radavononitch, G.: Ultrasonji atlas of nomal brain of infint. UJtrasound Med Biol 1: $759,1974$.

4. Johnson, H.L., Mack La. Rumack, C.M., Frost, M. Rashbaum, C.: B-Mode cchoencephalograplyy in the normal and high risk infant. Am J Radiol 133: 375, 1979.

5. Morgan, C.L., Thought, W.S., Rothman, S.J., Iiménez. J.P. Comparison of gray scale ultrasonoguphy and computed tomograpliy in the evaluation of macroctionia in infants. Radiology 132: 119, 1979.

6. Ben.Ora. Avi, Unda, E., Hatch, G., Solida, B.: The anterior fontanclle as an acoustic window to the nconatal ventricular system. J Clin U1trasound 8 : $65,1980$.

7. Allon, C. W., Roveto, A,C. Sawyer. I., R., Courtnel'. $S F_{\text {.: }}$ Sector scan ultrasound maging though the anterior fontanelle. Am J Dis Child 134: 1028. 1980.

8. Couture, A., Fertan, J.L., Blum, M., Montoya. F. German. M. Montova, P., Pous, J.G., Senac, J.P. Bonnet, $H .:$ La woup transfontanellaire en echots] céphalographic. Ann Radio] 23: 649, 1980.

9. Couture, A., Cadier, L.: Lislographie cérébrale par voic transfontancllaire Ed. Vigot, Paris. 1983: 3.

10. Jolnson, M.L. Dunne, M. G. Mack L.a. Rashboum, C.L.: Evaluation of fetal intracranial anatomy by static and real-time ultrasound. JCU 8: $311,1980$.

11. Pape, K.E., Cusick, G., Iouang, M.T.W., Blackwcll. R.I., Sheruyod, A., Thorburn, R.II., Reynolds. E.O.R.: Ultrasound detection of brain damage in pretern intant, Lancet $1 \cdot 1261,1979$.

12. Hober, $K$., wachter, R.D., Christenson, P.C., Vaucher, J., Sahn, D.J., Smith, J.R.: Lltrasonic craluation of intracranial pathology in infants: a new technique. Radiology 134: 173, 1980.

13. Gront, E.G., Schellinger, D., Borts, F.T. MacCultough, D.C., Friedman, G.R., Sivasubramanian. K.N. Smith, $Y$.: Real time sonography of the neonatal and infant head. AJR 136; 265, 1981.

14. Schlager, C.G.: Neuropediatría I., Santiaguehile. Andrés Bello, 1983, 39. 
15. Domergue, A.: Atlas d'échotomograhie cránioencéphalique du nourrizson normal. Confrontations échoanatomiques. Thése pour le Doctorat en Médecine, Montpellier, Juin 1980.

16. Shuman, W.P., Rogers, J.V., Mockl. A., Alvord, E.C., Christie, D.P.: Real time sonographic sector scanning of the neonatal cranium technique and normal anatomy. AJR 137: 821, 1981

17. Klein, $F, y$ cols. Lxploración ecográfica del encéfalo del recién nacido de término y de pretétmino, parámetros de normalidad. Rev Chil Obstet Ginecol 48: 255, 1983 .

18. Volpe, J.J.: Perinatal hypoxic ischemic brain injury. Pediatr Clin North Am 23: 383, 1976.

19. Pinro, F. y cols.: Ultrasonografía de cráneo. Su aporte al estudio del recién nacido asfixiado $y$ evolución posterior. Rev Chil Obstet Ginecol 50: 201,1985 .

20. Fedrick, J., Butler, N.R.: Certain causes of Neonatal death. II Intraventricular hacmorrhage. Biol Neonate 15: $257,1970$.

21. Hombleton, G., Wiggleworth, J.S.: Origin of intra venticular hemorrhage in the pertern infant. Arch Dis Child 51: 651, 1976.

22. Donat, J.F., Okazaki, H. Kleinberg, F., Reagan, $T . J_{.:}$Intraventricular liemorrhages in full term and premature infants. Mayo Clin Proc 53: 437, 1978.

23. Ahmann, P.A., Lazzara, A. Kykes, F.D., Brann. $A . W$. . Schwartz, J.F.: Intraventricular hemorrhage in the high risk preterm infant: incidence and outcome. Ann Neurol 7: 118,1980.

24. Dykes, F.D., Lazzara, A., Ahmann. P., Blumenstein, B., Schwartz, J., Brann, A.W.: Intraventricular hemorrhage: a prospective evaluation of etiopatho. genesis. Pediatrics 66: 42, 1980.

25. Norambuena, N. y cols.: Díagnóstico de la hemorragia intracraneana mediante ecografía encefálica en recién nacidos de bajo peso (menos de 1.500 grs.) Correlación anatomapatológica. Rev Chil Obstet Ginecol 48: 251, 1983 .

26. Allan, W.C., Roveto, C.A., Sawyer, L.R., Coutrney, S.E.: Sector scan ultrasound imaging through the anterior fontanel, Am J Dis Child 134: 1028, 1980.

27. Bejar, R., Curbelo, V., Coen. R.W., Leopold, G., James, H., Gluck, L,: Diagnosis and follow-up of intraventricular and intracerebral hemorrhage by ultrasound: studies of infant's brain through the fortanelles and sutures. Pediatrics 66: 661, 1980.

28. Cooke, R.W.I.: Letter to the edjtor, Lancet 3: 38 , 1979.

29. Johnson, M.L., Rumack, C.M., Mann-s, E.J. Appareti, K.E.: Detection of neonatal intracranial hemorrhage utilizing real-tione and static ultrasound, JCU 9: 427, 1981 .

30. Shankaran, S, Slovis, T.L., Bedar, M.P., Poland. R.I.: Sonographic classification of intracranial hemorrhage. A pronostic indicator of mortality, morbidity and shorterm neurologic outcome. J Pediati 100: 469, 1982.

31. Sauerbrei, E.E., Cooperberg, P.L.: Neonatal brain: sonography of congenital abrormalities. AJR 136: 1167,1981 .

32. Lavery, J.P., Kubarych, S.F., Queenan, J.T.: Neural tube defect discovered at routine ultrasound evaluation. JCU 8: $55,1980$.

33. Walter, C., Allan and Alistair, G.S., Philips,: Neonatal werebral pathology diagnosed by ultrasound. Clin Perinatol 12: 195, 1985.

34. Cunningham, M.E., Walls, W.I.: Litrasound in the evaluation of anencephaly. Radiology 118: 165 , 1976.

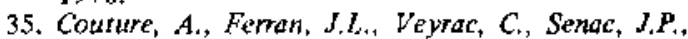
Germain, $H_{1}$ Monfoya, $F$. Bonnet, $H$.: Apport de l'échnencépahlographie transfontanellaire chez le nouveau-né. Arch Fr Pediatr 38: 481, 1981.

36. Fiske, C.E., Filly, R.A.: Ultrasound evaluation of the normal and abnormal fetal neural axis. Radiol Clin North Am 20: 285, 1982.

37. Hidalgo, H., Bowie, J., Rasenberg, E.R., Ram, P.C., Ford. $K$, Lipsit, E.: In utero sonographic diagnosis of fetal cerebral anormalies. AJR 139: 143, 1982.

38. Dunne, M.G., Johnson, M.L.: The ultrasonic demonstration of fetal abnormalities in utero. J Reprod Med 23: 195, 1979.

39. Hill, A., Shockelford, G.D., Volpe, J.J.: Ventriculitis with neonatal bacteria meningitis: identification by real time ultrasound. J Pediatr 9: 133. 1981.

40. Enzmenn, D.R., Britt, R.H., Lyon, B., Coroll, B., Witson, D.A., Buxfon. J.: High resolution ultrasound evaluation of experimental brain abscess evolution: comparison with computed tomography and neuropathology. Radiology 142: 95, 1982 . 